\begin{tabular}{|c|c|}
\hline \multicolumn{2}{|c|}{ Statistica Sinica Preprint No: SS-2020-0386 } \\
\hline Title & Identifiability of Bifactor Models \\
\hline Manuscript ID & SS-2020-0386 \\
\hline URL & http://www.stat.sinica.edu.tw/statistica/ \\
\hline DOI & $10.5705 /$ ss. 202020.0386 \\
\hline Complete List of Authors & $\begin{array}{l}\text { Guanhua Fang, } \\
\text { Jinxin Guo, } \\
\text { Xin Xu, } \\
\text { Zhiliang Ying and } \\
\text { Susu Zhang }\end{array}$ \\
\hline Corresponding Author & Susu Zhang \\
\hline E-mail & szhan105@illinois.edu \\
\hline Jotice: Accepted version subje & ct to English editing. \\
\hline
\end{tabular}


Statistica Sinica

\title{
Identifiability of Bifactor Models
}

\author{
Guanhua Fang ${ }^{1}$, Jinxin $\mathrm{Guo}^{2}$, Xin $\mathrm{Xu}^{2}$, Zhiliang Ying ${ }^{3}$ and Susu Zhang ${ }^{4}$ \\ ${ }^{1}$ Baidu Research ${ }^{2}$ Northeast Normal University \\ ${ }^{3}$ Columbia University, ${ }^{4}$ University of Illinois at Urbana-Champaign
}

Abstract: The bifactor model and its extensions are multidimensional latent variable models, under which each item measures up to one subdimension on top of the primary dimension(s). Despite their wide applications to educational and psychological assessments, this type of multidimensional latent variable models may suffer from non-identifiability, which can further lead to inconsistent parameter estimation and invalid inference. The current work provides a relatively complete characterization of identifiability for the linear and dichotomous bifactor models and the linear extended bifactor model with correlated subdimensions. In addition, similar results for the two-tier models are also developed. Illustrative examples are provided on checking model identifiability through inspecting the factor loading structure. Simulation studies are reported that examine estimation consistency when the identifiability conditions are/are not satisfied.

Key words and phrases: identifiability, bifactor model, educational and psychological measurement, two-tier model, item factor analysis, testlet, multidimensional item response theory 


\section{Introduction}

The bifactor method (Holzinger and Swineford, 1937) for factor analysis is a constrained factor analytic model, which assumes that the responses to a set of test items can be accounted for by $(G+1)$ uncorrelated latent dimensions, with one primary dimension assessed throughout the test and $G$ secondary "group" dimensions. It further constrains each item to have nonzero loading on only one of the $G$ secondary dimensions. While the bifactor method for factor analysis (henceforth referred to as the linear bifactor model) was originally developed for continuous indicators, it has been extended to bifactor item response models (e.g., Gibbons and Hedeker, 1992; Gibbons et al., 2007; Cai et al., 2011) for dichotomous, ordinal, or nominal item responses through the introduction of link functions, such as a probit link. The assumption of orthogonality among the secondary dimensions has also been relaxed in the extended bifactor model (e.g., Jennrich and Bentler, 2012; Jeon et al., 2013) to allow for covariance between secondary dimensions unexplained by the primary dimension. The bifactor model with one primary dimension has further been extended to the twotier model Cai, 2010) with $L \geq 1$ primary dimensions and $G$ secondary dimensions, with each item measuring up to one secondary dimension and the secondary dimensions being independent of the primary ones. 
The bifactor model and its extensions have demonstrated significant practical merits in educational and psychological assessments. Compared to uni- or low-dimensional latent trait models, they can accommodate the local dependence among clusters of items measuring the same subdimensions and produce subdimension trait estimates. Compared to general multidimensional latent variable models, they not only allow for the production of overall score(s) but also remarkably reduce the computational burden of high dimensional latent trait model estimation. The bifactor model and its extensions have hence been applied to hundreds of cognitive and psychological assessments, including psychiatric screenings that cover various domains of clinical disorders (e.g., Gibbons et al., 2009), personality instruments that tap on multiple facets of the same trait (e.g., Chen et al. 2012), intelligence batteries with multiple subscales (e.g., Gignac and Watkins, 2013), and patient-reported outcome measures with broad situational representations (e.g., Reise et al., 2007). In educational testing, the bifactor model and its variants have seen wide applications to assessments that involve testlets, that is, multiple questions originated from the same stem (e.g., passage; Bradlow et al., 1999; DeMars, 2006, 2012; Jeon et al., 2013; Rijmen, 2010). In longitudinal assessments with repeated administrations of the same item, bifactor and two-tier models can account for the within- 
person dependence of responses to the same item across time points (see, Cai et al., 2016). Parameter estimation for the bifactor and two-tier models have been implemented in many commercial and open-source statistical software programs. It is also worthwhile to note that bifactor model is robust in practice. It tends to fit any data set better than other confirmatory models regardless of the population's true models (Caspi et al. 2014). Thorough introductions to the bifactor model and its generalizations can be found in Reise (2012) and Cai et al. (2011).

Identifiability is a key issue in any type of latent variable modelling Allman et al., 2009: $\mathrm{Xu}$ and Zhang, 2016; Xu et al., 2017; $\mathrm{Gu}$ and $\mathrm{Xu}$, 2019; Chen et al., 2015, 2019). Intuitively, a model is identifiable if distinct parameter values produce unique probability distributions of observed responses. Model identifiability is a necessary condition for consistency of parameter estimation and valid statistical inference. Without any additional requirements, the bifactor model has been shown to be non-identifiable in previous studies: Under the linear bifactor model, Green and Yang (2018) showed that two distinct sets of model parameters could produce the same model-implied covariance matrix. Eid et al. (2018) showed that non-identifiability could arise in structural equation models with bifactor measurement model. The current paper addresses the identifiability issue of 
the bifactor model and its extensions by providing a relatively complete theory. We obtain the sufficient and necessary conditions for the identifiability of the standard bifactor model with both continuous and binary responses. Further, the necessary conditions for the extended bifactor model identifiability are given, and sufficient conditions for the identifiability of the extended bifactor and two-tier models are provided. For the dichotomous responses, discussions are limited to probit item response models, as the theoretical identifiability of logistic item response models with multivariatenormally distributed latent traits is a more complex issue. The identifiability of the aforementioned models can be achieved through the constraints on the loading structure of the items onto the general and specific dimensions. These results provide practitioners with viable means to examine identifiability of a certain test through a set of easily checkable conditions.

A number of simple rules for checking confirmatory factor model identification have been proposed in prior studies (e.g., Bollen, 1989, Reilly, 1995; Reilly and O'Brien, 1996). Among them, the $t$-rule, which requires the number of unknown model parameters to not exceed the number of unique covariance terms, provides a necessary but not sufficient condition for model identification. Another set of well-known rules are the 3-indicator and 2-indicator rules (see Bollen, 1989), which are sufficient for identifica- 
tion but require simple factor loading structure, thus not applicable to the bifactor models. Empirical tests for local identification based on the information or the Jacobian matrix have been implemented in factor model estimation programs such as LISREL (Jöreskog and Sörbom, 1993). However, local identifiability of parameters within the neighborhood of the estimates does not guarantee global identifiability in the entire parameter space. For many general factor models where no simple rule is applicable, checking global identification requires solving a system of equations for observed and model-implied means and covariances and determining whether the solution to each parameter is unique, either manually or with the aid of computer algebra systems (Bollen and Bauldry, 2010; Kenny and Milan, 2012). Thanks to the special structure of the bifactor model, simple and checkable sufficient and necessary conditions can be developed. Readers are referred to Bollen (1989), Bollen and Bauldry (2010) and Jöreskog and Sörbom (1993) for introductions to common methods for checking general factor model identification.

The rest of the paper is organized as follows. Sections 2 presents the identifiability results on the linear bifactor, extended bifactor, and two-tier models. Section 3 extends the theoretical results on the three models to dichotomous responses with the probit link. Section 4 discusses the con- 
nections between the new results and the existing literature on bifactor identifiability. A discussion of the findings is provided in Section 5. In the supplementary file, simulation studies are designed to verify the theoretical identifiability results by examining the estimation consistency under several identifiable and non-identifiable loading structures. Additionally, the supplementary contains proofs of the main theoretical results and more detailed examples.

\section{Linear Bifactor Model and Extensions}

The current section presents results on the identifiability of the linear standard bifactor, linear extended bifactor, and linear two-tier models, which assume the response to each item to be normally distributed, with mean equal to an intercept plus a linear combination of the latent factor scores. This class of models is thus suitable for continuous observed indicators.

It is worth introducing the concept of identifiability in general before moving on to specific models. In mathematical terms, a statistical model may be specified by a pair $(\mathcal{S}, \mathcal{P})$, where $\mathcal{S}$ is the set of possible observations or the sample space, and $\mathcal{P}$ is a set of probability distributions on $\mathcal{S}$, which is parameterized as $\mathcal{P}=\left\{P_{\theta}, \theta \in \Theta\right\}$. The set $\Theta$ defines the parameter space. We say the model parameter $\theta^{*}$ is identifiable (or model is identifiable at 
$\left.\theta^{*}\right)$ if $\mathcal{P}_{\theta}(y)=\mathcal{P}_{\theta^{*}}(y)$ for all $y \in \mathcal{S}$ implies $\theta=\theta^{*}$. Essentially, identifiability implies that the underlying distribution of the observed data cannot admit two distinct sets of parameter values.

\subsection{Standard bifactor model}

The standard bifactor model (Holzinger and Swineford, 1937) assumes that the response to each item in a test can be explained by one general factor, which runs through the test, and up to one group factor, which runs through a subset of items. Without loss of generality, we refer to a subset of items which load on the same group factor as a testlet (e.g., Bradlow et al., 1999: DeMars, 2006), but the model formulation is equally applicable to psychological assessments with subdimensions and cognitive batteries with subtests. Specifically, consider a test with $J$ items which can be partitioned into $G$ testlets, where the $g$ th testlet consists of $J_{g}$ items and $\sum_{g} J_{g}=J$. Let $\mathcal{B}_{g}$ denote the set of items in the $g$ th testlet. Under the standard linear bifactor model, the response to item $j \in\{1, \ldots, J\}$ in testlet $g_{j} \in$ $\{1, \ldots, G\}, Y_{j}$, is given by

$$
Y_{j}=d_{j}+a_{j 0} \eta_{0}+\sum_{g=1}^{G} a_{j g} \eta_{g}+\epsilon_{j}
$$

where $\eta_{0}$ is the respondent's latent score on the general factor, $\eta_{g}$ is the latent score on the $g$ th group factor, $a_{j k}$ is item $j$ 's loading on the $k$ th latent 
2.1 Standard bifactor model

dimension, $d_{j}$ is the item intercept, and $\epsilon_{j}$ is the random error unexplained by the latent factors. Across all items, the $\epsilon_{j}$ s are assumed to be independently distributed with mean 0 and variance $\lambda_{j}$, that is, $\epsilon_{j} \sim N\left(0, \lambda_{j}\right)$. Further, for item $j$ in testlet $g_{j}$, it is assumed that $a_{j g}=0$ for all $g \neq g_{j}$, in other words, the loadings of item $j$ on all other group factors are restricted to 0. Thus, Equation (2.1) simplifies to

$$
Y_{j}=d_{j}+a_{j 0} \eta_{0}+a_{j g_{j}} \eta_{g_{j}}+\epsilon_{j}
$$

Let $\boldsymbol{\eta}=\left(\eta_{0}, \eta_{1}, \ldots, \eta_{G}\right)^{T}$ denote the vector of latent traits of a respondent, which is assumed to follow the multivariate normal distribution with zero mean and covariance $\Sigma$, that is

$$
\boldsymbol{\eta} \sim \operatorname{MVN}(\mathbf{0}, \boldsymbol{\Sigma})
$$

The standard bifactor model further assumes that all general and group factors are independent, in other words, $\boldsymbol{\Sigma}=\boldsymbol{I}_{(1+G),(I+G)}$, where $\boldsymbol{I}_{(1+G),(I+G)}$ is the $(1+G) \times(1+G)$ identity matrix. The mean and standard deviation of each latent factor are fixed to 0 and 1, respectively, to resolve the location and scale indeterminacy of the latent dimensions.

Let $\boldsymbol{A}=\left[\mathbf{a}_{0}, \mathbf{a}_{1}, \ldots, \mathbf{a}_{G}\right]$ denote the $J \times(1+G)$ matrix of factor loadings, where the first column $\mathbf{a}_{0}=\left(a_{10}, \ldots, a_{J 0}\right)^{T}$ is the items' loadings on the general factor, and the subsequent $G$ columns $\left(\mathbf{a}_{1}, \ldots, \mathbf{a}_{G}\right)$ are the 


\subsection{Standard bifactor model}

loadings on each of the $G$ testlets. Note that $\boldsymbol{A}$ is a sparse matrix, with most of the testlet-specific loadings restricted to zero by the single group factor loading assumption. Let $\mathbf{d}=\left(d_{1}, \ldots, d_{J}\right)^{T}$ denote the length- $J$ vector of item intercepts, and let $\boldsymbol{\lambda}=\left(\lambda_{1}, \ldots, \lambda_{J}\right)$ denote the vector of item unique variances. To resolve the sign indeterminacy of the latent factors, we further assume without loss of generality that for each factor, the first item that loads on the factor has positive loading. Under the standard bifactor model, the parameter space $\mathfrak{P}$ is then given by $\mathfrak{P}=$ $\{(A, \mathbf{d}, \boldsymbol{\lambda}) \mid$ The sign of first non-zero element in every column of $A$ is positive $\}$. The definition of identifiability of the standard bifactor model is as follows.

Definition 1. We say a linear bifactor model is identifiable at $(A, \mathbf{d}, \boldsymbol{\lambda})$ if, for any other set of parameter $\left(A^{\prime}, \mathbf{d}^{\prime}, \boldsymbol{\lambda}^{\prime}\right)$ that defines the same probability distribution, it must hold that

$$
(A, \mathbf{d}, \boldsymbol{\lambda})=\left(A^{\prime}, \mathbf{d}^{\prime}, \boldsymbol{\lambda}^{\prime}\right)
$$

Before stating the identifiability results on the standard bifactor model, we introduce some additional notation. Let $\bar{A}_{g}$ be the submatrix of $A$ corresponding to items in testlet $g$ (i.e., $\left.B_{g}\right)$, that is, $\bar{A}_{g}=\left(\mathbf{a}_{0}\left[\mathcal{B}_{g}\right], \mathbf{a}_{g}\left[\mathcal{B}_{g}\right]\right)$, where $x\left[\mathcal{B}_{g}\right]$ denotes of subvector with entries in set $\mathcal{B}_{g}$. Further, let $A[\mathcal{B},:]$ denote the submatrix of $A$ consisting only of rows in some generic set $\mathcal{B}$. We define the following subsets. 


\subsection{Standard bifactor model}

- $\mathcal{H}_{1}=\left\{g \mid \mathbf{a}_{0}\left[\mathcal{B}_{g}\right] \neq \mathbf{0}\right\}$ : the set of testlets in $\{1, \ldots, \mathrm{G}\}$ with nonvanishing main factors, that is, at least one item in the testlet has nonzero true loading on the main dimension, $\eta_{0}$;

- $\mathcal{Q}_{g}=\left\{j \mid \mathbf{a}_{g}[j] \neq 0\right\}$ : the set of items in the $g$ th testlet with nonzero true loadings on the testlet-specific factor;

- $\mathcal{H}_{2}=\left\{g \mid\right.$ there exists a partition of $\mathcal{B}_{g}$, i.e., $\mathcal{B}_{g}=\mathcal{B}_{g, 1} \cup \mathcal{B}_{g, 2}, \mathcal{B}_{g, 1} \cap$ $\mathcal{B}_{g, 2}=\emptyset$, such that $\bar{A}_{g}\left[\mathcal{B}_{g, 1},:\right], \bar{A}_{g}\left[\mathcal{B}_{g, 2},:\right]$ are of full column rank. $\}:$ the set of testlets that can be partitioned into two disjoint subsets of items, where each submatrix of (main and testlet) factor loadings has full column rank.

The following theorem characterizes the sufficient and necessary conditions for the identifiability of the linear standard bifactor model.

Theorem 1. Under the standard linear bifactor model, the model parameters are identifiable if and only if it satisfies one of the following conditions:

P1 $\left|\mathcal{Q}_{g}\right| \geq 3$ for all $g=1, \ldots, G ;\left|\mathcal{H}_{1}\right| \geq 3$.

P2 $\left|\mathcal{Q}_{g}\right| \geq 3$ for all $g=1, \ldots, G ;\left|\mathcal{H}_{1}\right|=2 ;\left|\mathcal{H}_{2}\right| \geq 1$.

Theorem 1 gives the minimum requirements for identifiability of the standard bifactor model. Specifically, it requires the test to contain at least 
2.2 Extended bifactor model

2 testlets, each containing at least 3 items. In addition, if there are only 2 testlets, Theorem 1 requires that one of them can be partitioned into 2 disjoint subsets of items, such that both subsets have linearly independent primary and testlet-specific factor loadings.

\subsection{Extended bifactor model}

The extended bifactor model, also known as the oblique bifactor model (Jennrich and Bentler, 2012), relaxes the assumption of independence between the secondary dimensions. Instead of restricting the latent covariance matrix, $\Sigma$, to be the identity matrix, the extended bifactor model allows the covariance between the latent dimensions to take the form of $\boldsymbol{\Sigma}=\left(\begin{array}{cc}1 & \mathbf{0}^{T} \\ \mathbf{0}^{T} & \boldsymbol{\Sigma}_{G}\end{array}\right)$, where the covariance matrix for the testlet dimensions, $\Sigma_{G}$, is positive-definite with all diagonal elements being 1 and no additional restriction on off diagonal elements. Note that the covariances between the primary dimension and each testlet dimension is still restricted to be 0 .

Under the extended bifactor model, in addition to the item intercepts, loadings, and unique variances, the latent covariance matrix also needs to be estimated. Let $\mathfrak{P}=\left\{\left(A, \mathbf{d}, \Sigma_{G}, \boldsymbol{\lambda}\right) \mid\right.$ first non-zero element in every column of $A$ is positive, $\operatorname{diag}\left(\Sigma_{G}\right)=1, \Sigma_{G}$ positive definite $\}$ denote the parameter space of the extended bifactor model. Then the identifiability of the ex- 
tended bifactor model is defined as follows.

Definition 2. We say a linear extended bifactor model is identifiable at $\left(A, \mathbf{d}, \Sigma_{G}, \boldsymbol{\lambda}\right)$ if for any other set of parameters $\left(A^{\prime}, \mathbf{d}^{\prime}, \Sigma_{G}^{\prime}, \boldsymbol{\lambda}^{\prime}\right)$ that defines the same probability distribution,

$$
\left(A, \mathbf{d}, \Sigma_{G}, \boldsymbol{\lambda}\right)=\left(A^{\prime}, \mathbf{d}^{\prime}, \Sigma_{G}^{\prime}, \boldsymbol{\lambda}^{\prime}\right)
$$

must hold.

In addition to $\mathcal{H}_{2}$, and $\mathcal{Q}_{g}$ in the standard bifactor model identifiability results, we introduce another set that is key to the identifiability of the extended bifactor model:

- $\mathcal{H}_{3}=\left\{g \mid \bar{A}_{g}\right.$ has column rank 2$\}$ : the set of testlets with full main factor and testlet-specific factor information, that is, columns $\mathbf{a}_{0}\left[\mathcal{B}_{g}\right]$ and $\mathbf{a}_{g}\left[\mathcal{B}_{g}\right]$ are linearly independent.

Theorem 2 gives two sets of sufficient conditions for identifiability of the extended linear bifactor model.

Theorem 2. Under the linear extended bifactor model, the model parameters are identifiable if one of the following sets of requirements is satisfied:

E1S $\left|\mathcal{Q}_{g}\right| \geq 3$ for all $g=1, \ldots, G ;\left|\mathcal{H}_{3}\right| \geq 3$.

E2S $\left|\mathcal{Q}_{g}\right| \geq 3$ for all $g=1, \ldots, G ;\left|\mathcal{H}_{3}\right|=2 ;\left|\mathcal{H}_{2}\right| \geq 1$. 
2.2 Extended bifactor model

The sufficient conditions in Theorem 2 are very similar to the sufficient and necessary conditions for the standard bifactor model in Theorem 1 , where $\mathcal{H}_{3}$ is the counterpart to $\mathcal{H}_{1}$. Unlike $\mathcal{H}_{1}$, which contains testlets with nonzero main-factor loading vector, $\mathcal{H}_{3}$ further requires the main- and testlet-factor loading vectors to be linearly independent. Note that E1S and E2S are sufficient for identifiability of the extended bifactor model but they are not necessary. Theorem 3 provides the necessary but not sufficient conditions, that is, the minimum conditions that need to be met.

Theorem 3. Under the linear extended bifactor model, the model parameters are identifiable only if both conditions below are satisfied.

E1N $\left|\mathcal{Q}_{g}\right| \geq 2$ for all $g=1, \ldots, G ;$ and $\left|\mathcal{Q}_{g}\right| \geq 3$ for $g$ such that $\Sigma_{G}[g,-g]=$ 0. E2N $\left|\mathcal{H}_{3}\right| \geq 2$.

Essentially, at least two testlets with linearly independent main- and testlet-factor loadings are required. In addition, each subdimension should be measured by at least 2 items, and if a subdimension is uncorrelated with others, at least 3 items are required as before. The nonzero correlation between the testlet factors provides additional information on the testletspecific loadings, reducing the number of required items per testlet to 2 for 
2.2 Extended bifactor model

those testlets with nonzero correlations with others.

Note that $E 1 N$ and E2N together are not enough for the identifiability of extended bifactor model. Additional requirements are needed to render parameter identifiability. Theorem 2 gives one way to impose such additional requirements. The requirement of $\left|\mathcal{H}_{2}\right| \geq 1$ in E2S may be replaced by other requirements. See the following proposition.

Proposition 1. Under the linear extended bifactor model, the model parameters are identifiable if $E 1 N$ and $E 2 N$ are satisfied, and

E3S There exists $g_{1}, g_{2} \in \mathcal{H}_{3}$, such that (1) $\Sigma_{G}\left[g_{1}, g_{2}\right] \neq 0$; (2) $\left|\mathcal{Q}_{g_{1}}\right| \geq$ $3,\left|\mathcal{Q}_{g_{2}}\right| \geq 3$; and (3) Kruskal rank of $\bar{A}_{g_{1}}^{T}$ is 2. (A matrix A has Kruskal rank (Kruskal, 1977) $R$ if any $R$ columns of $A$ are linearly independent).

Remark 1. The gap between the necessary and sufficient conditions in Theorems 2 and 3 is that $\left|\mathcal{H}_{3}\right| \geq 2$ itself cannot guarantee identifiability. Either more testlets $\left(\left|\mathcal{H}_{3}\right| \geq 3\right)$ are needed or at least one of the testlets needs to be "strong" $\left(\left|\mathcal{H}_{2}\right| \geq 1\right)$. Non-zero correlations $(\Sigma)$ between testlet factors increase the complexity of the identifiability problem compared to the uncorrelated case.

Remark 2. Conditions in Theorems 1- 3 are easy to check in practice. We only need to do simple algebras (i.e. counting the number of non- 
zero entries, computing the column rank, etc.) on the estimated loading matrix $A$ and covariance matrix $\Sigma$. Additionally, $\mathcal{H}_{2} \geq 1$ in Condition P2 generically holds when a testlet contains four or more items.

Example 1. Consider an extended bifactor model with three testlets, where testlet 1 has only two items. Suppose the true $A$ and $\Sigma_{G}$ are given by

$$
A=\left(\begin{array}{cccc}
a_{10} & a_{11} & 0 & 0 \\
a_{20} & a_{21} & 0 & 0 \\
a_{30} & 0 & a_{32} & 0 \\
a_{40} & 0 & a_{42} & 0 \\
a_{50} & 0 & a_{52} & 0 \\
a_{60} & 0 & a_{62} & 0 \\
a_{70} & 0 & 0 & a_{73} \\
a_{80} & 0 & 0 & a_{83} \\
a_{90} & 0 & 0 & a_{93}
\end{array}\right), \quad \Sigma_{G}=\left(\begin{array}{ccc}
1 & \sigma_{12} & \sigma_{13} \\
\sigma_{12} & 1 & \sigma_{23} \\
\sigma_{13} & \sigma_{23} & 1
\end{array}\right)
$$

where $\sigma_{12}, \sigma_{13}, \sigma_{23} \neq 0$, any $A_{g}(g=1,2,3)$ has 2 linearly independent columns. According to Proposition 1, the model parameter is identifiable, even though $\left|\mathcal{Q}_{1}\right|=2$. 


\subsection{Two-tier model}

The two-tier model Cai, 2010) extends the standard bifactor model by allowing for more than one primary dimensions. Consider a test that measures $L$ primary factors and $G$ group factors. Under the two-tier model, denote latent factors by $\boldsymbol{\eta}=\left(\boldsymbol{\eta}_{1}, \boldsymbol{\eta}_{2}\right)$ with $\boldsymbol{\eta}_{1}=\left(\eta_{1}, \ldots, \eta_{L}\right)^{T}$ and $\boldsymbol{\eta}_{2}=$ $\left(\eta_{L+1}, \ldots, \eta_{L+G}\right)^{T}$. The response to the $j$ th item in testlet $g_{j}$ is given by

$$
Y_{j}=d_{j}+\sum_{l=1}^{L} a_{j l} \eta_{l}+\sum_{g=L+1}^{L+G} a_{j g} \eta_{g}+\epsilon_{j} .
$$

Similar to the bifactor model, $\epsilon_{j}$ s are indepdent and normally distributed with $\epsilon_{j} \sim N\left(0, \lambda_{j}\right)$, and only $g_{j}$ th testlet factor loading is nonzero for item $j$, i.e., $a_{j g}=0$ for all $g \neq g_{j}$. The latent covariance matrix of $\boldsymbol{\eta}$ takes the form of $\left(\begin{array}{cc}\Sigma_{L} & \mathbf{0}^{T} \\ \mathbf{0}^{T} & I_{G \times G}\end{array}\right)$, where $\Sigma_{L}$ is a $L \times L$ positive-definite matrix with diagonal elements of 1 and no additional restriction on off-diagonal elements. Let $\mathfrak{P}=\left\{\left(A, \mathbf{d}, \Sigma_{L}, \boldsymbol{\lambda}\right) \mid\right.$ first non-zero element in every column of $A$ is positive, $\operatorname{diag}\left(\Sigma_{L}\right)=1, \Sigma_{L}$ positive definite $\}$ represent the model space of the twotier model. Further, let I denote the configuration mapping function, with $\mathbf{I}(X)=\tilde{X}$ where $\tilde{X}_{i j}=\mathbf{1}\left\{X_{i j} \neq 0\right\}$ for an arbitrary matrix $X$. The definition of two-tier model identifiability is stated as follows.

Definition 3. A linear two-tier model is identifiable at $\left(A, \mathbf{d}, \Sigma_{L}, \boldsymbol{\lambda}\right)$ if for any other set of parameters $\left(A^{\prime}, \mathbf{d}^{\prime}, \Sigma_{L}^{\prime}, \boldsymbol{\lambda}^{\prime}\right)$ that define the same distribution 
and satisfies $\mathbf{I}(A)=\mathbf{I}\left(A^{\prime}\right)$, it must hold that $\left(A, \mathbf{d}, \Sigma_{L}, \boldsymbol{\lambda}\right)=\left(A^{\prime}, \mathbf{d}^{\prime}, \Sigma_{L}^{\prime}, \boldsymbol{\lambda}^{\prime}\right)$.

Here, notice that a factor model is only identifiable up to some rotation. In the definition, the requirement for equal factor loading configurations, $\mathbf{I}\left(A^{\prime}\right)=\mathbf{I}(A)$, is put in place to resolve the rotational indeterminacy. We would like to point out that the identifiability of two-tier model is nontrivial, in the sense that the model could fail to be identifiable, even if (1) the loading matrix of main factors, $A_{:, 1: L}$, satisfies the usual identifiability conditions for multivariate factor models and (2) the testlets satisfy the identifiability conditions for the bifactor model. See the examples provided in the supplementary.

The proposed sufficient conditions for two-tier model identifiability build upon the sufficient conditions for unique variance identifiability under factor models with uncorrelated errors, which can be found in Theorem 5.1 of Anderson and Rubin (1956) and is rephrased below.

Theorem 4. (Anderson and Rubin, 1956). Consider a general factor model with implied covariance matrix $\Psi=A \Sigma A^{T}+\Lambda$, where the item error covariance matrix, $\Lambda$, is diagonal with $\operatorname{diag}(\Lambda)=\boldsymbol{\lambda}$. Then $A \Sigma A^{T}$ and $\Lambda$ are identifiable if the following holds:

C0 If any row of $A$ is deleted, there remain two disjoint submatrices of $A$ with full column rank. 
Under the two-tier model, let $\bar{A}_{g}$ be the submatrix of $A$ corresponding to items in testlet $g$, that is, $\bar{A}_{g}=\left(A\left[\mathcal{B}_{g}, 1: L\right], A\left[\mathcal{B}_{g}, L+g\right]\right)$. Similarly, for a subset of testlets $\mathcal{G}_{1} \subseteq\{1, \ldots, G\}$, denote the submatrix of $A$ corresponding to testlets in $\mathcal{G}_{1}$ by $\bar{A}_{\mathcal{G}_{1}}=\left(A\left[\mathcal{B}_{\mathcal{G}_{1}}, 1: L\right], A\left[\mathcal{B}_{\mathcal{G}_{1}}, L+\mathcal{G}_{1}\right]\right)$, where $\mathcal{B}_{\mathcal{G}_{1}}=$ $\bigcup_{g \in G_{1}} \mathcal{B}_{g}$ and $L+\mathcal{G}_{1}=\left\{L+g^{*} \mid g^{*} \in \mathcal{G}_{1}\right\}$. We introduce two sets, $\mathcal{H}_{4}$ and $\mathcal{H}_{5}$, that are essential to the identifiability results for the two-tier model:

- $\mathcal{H}_{4}=\left\{g \mid A\left[\mathcal{B}_{g}, 1: L\right]\right.$ is of full column rank $\}$ : the set of testlets with non-degenerate main factor information, that is, the $L$ columns corresponding to the main factor loadings are linearly independent;

- $\mathcal{H}_{5}=\left\{g \mid \bar{A}_{g}\right.$ is of full column rank $\}$ : the set of testlets with nondegenerate all-factor information, that is, with linearly independent main factor and testlet factor loadings.

The following theorem provides the sufficient conditions for identifiability of the linear two-tier model.

Theorem 5. Under the linear two-tier model, if the true loading matrix, $A$, satisfies Condition CO and one of Conditions T1S - T3S, then the parameters are identifiable.

T1S $\left|\mathcal{H}_{4}\right| \geq 3, A\left[\mathcal{B}_{\mathcal{H}_{4}}, 1: L\right]$ contains an identity, where $\mathcal{B}_{\mathcal{H}_{4}}$ is the set of items that makes up the testlets in $\mathcal{H}_{4}$. 
T2S $\left|\mathcal{H}_{4}\right| \geq 2,\left|\mathcal{H}_{5}\right| \geq 1, A\left[\mathcal{B}_{\mathcal{H}_{4}}, 1: L\right]$ contains an identity.

T3S A[:,1:L] contains an identity, and there exists a partition of testlets $\{1, \ldots, G\}=\mathcal{G}_{1} \dot{\cup} \mathcal{G}_{2}$, such that (1) $\bar{A}_{\mathcal{G}_{1}}$ has full-column rank, and (2) $A\left[\mathcal{B}_{\mathcal{G}_{2}}, 1: L\right]$ has full-column rank.

In other words, the linear two-tier model is identifiable if the following two conditions are simultaneously met:

- Removing any row of the loading matrix $A$, the remaining rows of $A$ can be partitioned into two disjoint submatrices, both of which contain $L+G$ linearly independent columns.

- One of the following is satisfied:

$T 1 S$ : (1) The test contains at least 3 testlets with linearly independent main factor loadings, and (2) within these testlets satisfying (1), for each main factor, there exists at least one item that exclusively measures this main factor (i.e., having nonzero loadings only on this main factor and possibly the testlet factor);

T2S : (1) The test contains 2 testlets with linearly independent main factor loadings, (2) within these testlets satisfying (1), for each main factor, there exists at least one item that exclusively mea- 
sures this main factor, and (3) at least one of the testlets satisfying (1) has linearly independent main and testlet factor loadings;

T3S : (1) For each main factor, at least one item in the test exclusively measures that main factor (aside from the testlet factor), and (2) the set of all testlets can be partitioned to two disjoint subsets, $\mathcal{G}_{1}$ and $\mathcal{G}_{2}$, such that (a) the loading matrix corresponding to the first subset of testlets, $\bar{A}_{\mathcal{G}_{1}}$, has $L+\left|G_{1}\right|$ linearly independent columns, and (b) for the second subset, the columns of main factor loadings, $A\left[\mathcal{B}_{\mathcal{G}_{2}}, 1: L\right]$, are linearly independent.

\section{Extensions to Dichotomous Responses}

Notwithstanding the wide application of linear factor models in the social science literature, a large proportion of educational and psychological assessments consist of items with dichotomous responses, for instance, cognitive questions where an examinee responds either correctly (1) or incorrectly (0), or clinical screening questions where a participant either exhibits certain behavior (1) or not (0). Probit item response models, also known as normal ogive models (e.g., Thurstone, 1927; Lawley, 1943; Lord, 1952; Christoffersson, 1975), have been widely adopted for dichotomous responses. In general, consider a test of $J$ items. The responses to the $J$ 
items are assumed to be locally independent given the respondents' latent traits, $\boldsymbol{\eta}$, and the probability of responding "1" to the $j$ th item is given by

$$
P\left(Y_{j}=1 \mid \boldsymbol{\eta}\right)=P_{\epsilon}\left(\epsilon_{j} \leq d_{j}+\mathbf{a}_{j}^{T} \boldsymbol{\eta}\right)=\Phi\left(d_{j}+\mathbf{a}_{j}^{T} \boldsymbol{\eta}\right),
$$

where $\epsilon_{j} \sim N(0,1), \Phi(\cdot)$ is the standard normal cumulative distribution function (i.e., the probit link), and $\boldsymbol{\eta} \sim N(0, \Sigma), d_{j}, \mathbf{a}_{j}$ are the person latent traits, item intercept, and item slopes/loadings, respectively. Similar as before, let $A$ denote the matrix of factor loadings.

Item bifactor model and extensions have also been proposed to accommodate for dichotomous response tests with underlying bifactor-like latent structures. Examples of such models include the item bifactor model Gibbons and Hedeker, 1992), the extended item bifactor model (Jeon et al., 2013), and the two-tier item factor model (Cai, 2010). The identifiability results for the linear bifactor model and extensions do not directly apply to dichotomous item bifactor-type models due to different parameterizations and form of observed data. The current section presents the results on identifiability of the dichotomous bifactor model, extended bifactor model, and two-tier model with probit links.

Before introducing the identifiability conditions for each of the specific models, it is worth mentioning a few identities on the items' 1st, 2nd, and $k$ th moments implied by the general probit item factor model in (3.8), as 
well as their relationships with the thresholds and tetrachoric correlations (Pearson, 1900) under the probit model. Unlike linear models, we do not directly observe the mean and covariance matrix implied by the linear component (i.e., $d_{j}+\mathbf{a}_{j}^{T} \boldsymbol{\eta}_{j}$ ) under the probit model, but the threshold and tetrachoric correlations can be identified (Kendall and Stuart, 1958) and estimated with various approximation methods (e.g., Castellan, 1966; Olsson, 1979). In the following, we explain how the tetrachoric correlations relate to the idenfiability problem.

Let $\xi$ denote a standard normal random variable. Note that, at the population level (i.e., for a randomly chosen $\boldsymbol{\eta} \sim M V N(0, \Sigma)$ ), the marginal probability of observing a response of 1 on item $j$ is given by

$$
\begin{aligned}
P\left(Y_{j}=1\right) & =\mathbb{E}_{\boldsymbol{\eta}} P\left(Y_{j}=1 \mid \boldsymbol{\eta}\right)=\mathbb{E}_{\boldsymbol{\eta}} \mathbb{E}_{\epsilon_{j}}\left[\mathbf{1}\left\{\epsilon_{j} \leq d_{j}+\mathbf{a}_{j}^{T} \boldsymbol{\eta}\right\} \mid \boldsymbol{\eta}\right]=P\left(\epsilon_{j} \leq d_{j}+\mathbf{a}_{j}^{T} \boldsymbol{\eta}\right) \\
& =P\left(d_{j}+\sqrt{\mathbf{a}_{j}^{T} \Sigma \mathbf{a}_{j}+1} \xi \geq 0\right)=1-\Phi\left(-\frac{d_{j}}{\sqrt{\mathbf{a}_{j}^{T} \Sigma \mathbf{a}_{j}+1}}\right)
\end{aligned}
$$

And the probability that the responses to items $j_{1}$ and $j_{2}$ are both 1 is

$$
\begin{aligned}
& P\left(Y_{j_{1}}=1, Y_{j_{2}}=1\right)=\mathbb{E}_{\boldsymbol{\eta}} P\left(Y_{j_{1}}=1, Y_{j_{2}}=1 \mid \boldsymbol{\eta}\right) \\
= & P\left(\epsilon_{j_{1}} \leq d_{j_{1}}+\mathbf{a}_{j_{1}}^{T} \boldsymbol{\eta}, \epsilon_{j_{2}} \leq d_{j_{2}}+\mathbf{a}_{j_{2}}^{T} \boldsymbol{\eta}\right) \\
= & P\left(d_{j_{1}}+\sqrt{\mathbf{a}_{j_{1}}^{T} \Sigma \mathbf{a}_{j_{1}}+1} \xi_{j_{1}} \geq 0, d_{j_{2}}+\sqrt{\mathbf{a}_{j_{2}}^{T} \Sigma \mathbf{a}_{j_{2}}+1} \xi_{j_{2}} \geq 0\right) \\
= & \Phi_{2}\left(-\frac{d_{j_{1}}}{\sqrt{\mathbf{a}_{j_{1}}^{T} \Sigma \mathbf{a}_{j_{1}}+1}},-\frac{d_{j_{2}}}{\sqrt{\mathbf{a}_{j_{2}}^{T} \Sigma \mathbf{a}_{j_{2}}+1}}, \frac{\mathbf{a}_{j_{1}}^{T} \Sigma \mathbf{a}_{j_{2}}}{\sqrt{\mathbf{a}_{j_{1}}^{T} \Sigma \mathbf{a}_{j_{1}}+1} \sqrt{\mathbf{a}_{j_{2}}^{T} \Sigma \mathbf{a}_{j_{2}}+1}}\right),
\end{aligned}
$$


where $\Phi_{2}(a, b, \rho)=\mathbb{E}\left(X_{1} \geq a, X_{2} \geq b\right), X_{1}, X_{2} \sim N(0,1)$ and $\operatorname{corr}\left(X_{1}, X_{2}\right)=$ $\rho$. The $a, b$ and $\rho$ are commonly referred to as the thresholds and tetrachoric correlation under the probit framework. The probability for responding 1 simultaneously on $k$ items $\left(j_{1}, \ldots, j_{k}\right)$ is given by

$$
\begin{aligned}
& P\left(Y_{j_{1}}=1, \ldots, Y_{j_{k}}=1\right)=\mathbb{E}_{\boldsymbol{\eta}} P\left(Y_{j_{1}}=1, \ldots Y_{j_{k}}=1 \mid \boldsymbol{\eta}\right) \\
= & P\left(\epsilon_{j_{1}} \leq d_{j_{1}}+\mathbf{a}_{j_{1}}^{T} \boldsymbol{\eta}, \ldots, \epsilon_{j_{k}} \leq d_{j_{k}}+\mathbf{a}_{j_{k}}^{T} \boldsymbol{\eta}\right) \\
= & P\left(d_{j_{1}}+\sqrt{\mathbf{a}_{j_{1}}^{T} \Sigma \mathbf{a}_{j_{1}}+1} \xi_{j_{1}} \geq 0, \ldots, d_{j_{k}}+\sqrt{\mathbf{a}_{j_{k}}^{T} \Sigma \mathbf{a}_{j_{k}}+1} \xi_{j_{k}} \geq 0\right) \\
= & \Phi_{k}\left(-\frac{d_{j_{1}}}{\sqrt{\mathbf{a}_{j_{1}}^{T} \Sigma \mathbf{a}_{j_{1}}+1}}, \ldots,-\frac{d_{j_{2}}}{\sqrt{\mathbf{a}_{j_{2}}^{T} \Sigma \mathbf{a}_{j_{2}}+1}}, C_{\rho}\right)
\end{aligned}
$$

with tetrachoric correlation matrix $C_{\rho}\left[j_{1}, j_{2}\right]=\frac{\mathbf{a}_{j_{1}}^{T} \Sigma \mathbf{a}_{j_{2}}}{\sqrt{\mathbf{a}_{j_{1}}^{T} \Sigma \mathbf{a}_{j_{1}}+1} \sqrt{\mathbf{a}_{j_{2}}^{T} \Sigma \mathbf{a}_{j_{2}}+1}}$ and $C_{\rho}[j, j]=1$. Here $\Phi_{k}\left(a_{1}, \ldots, a_{k}, C_{\rho}\right)=\mathbb{E}\left(X_{1} \geq a_{1}, X_{k} \geq a_{k}\right), X_{1}, \ldots, X_{k} \sim$ $N(0,1)$ and $\operatorname{corr}\left(X_{k_{1}}, X_{k_{2}}\right)=C_{\rho}\left[k_{1}, k_{2}\right]$.

In the following, we show that threshold and tetrachoric correlations provide full information on probit binary item responses.

Proposition 2. Two sets of parameters define the same model if and only if their thresholds and tetrachoric correlations are equal, i.e.,

$$
\frac{d_{j}}{\sqrt{\mathbf{a}_{j}^{T} \Sigma \mathbf{a}_{j}+1}}=\frac{d_{j}^{\prime}}{\sqrt{\left(\mathbf{a}_{j}^{\prime}\right)^{T} \Sigma^{\prime} \mathbf{a}_{j}^{\prime}+1}} \forall j
$$

and

$\frac{\mathbf{a}_{j_{1}}^{T} \Sigma \mathbf{a}_{j_{2}}}{\sqrt{\mathbf{a}_{j_{1}}^{T} \Sigma \mathbf{a}_{j_{1}}+1} \sqrt{\mathbf{a}_{j_{2}}^{T} \Sigma \mathbf{a}_{j_{2}}+1}}=\frac{\left(\mathbf{a}_{j_{1}}^{\prime}\right)^{T} \Sigma^{\prime} \mathbf{a}_{j_{2}}^{\prime}}{\sqrt{\left(\mathbf{a}_{j_{1}}^{\prime}\right)^{T} \Sigma^{\prime} \mathbf{a}_{j_{1}}^{\prime}+1} \sqrt{\left(\mathbf{a}_{j_{2}}^{\prime}\right)^{T} \Sigma^{\prime} \mathbf{a}_{j_{2}}^{\prime}+1}} \forall j_{1} \neq j_{2}$ 


\subsection{Standard bifactor model}

It follows from the above proposition that checking the identifiability of probit bifactor models comes down to checking whether the probit threshold and tetrachoric correlations admit only one set of parameters. In other words, the probit bifactor models can be identified if $\left(d_{j}, \mathbf{a}_{j}\right)$ can be identified based on the thresholds (i.e., $\left.d_{j} /\left(\mathbf{a}_{j}^{T} \Sigma \mathbf{a}_{j}+1\right)^{1 / 2}, \forall j\right)$ and the pairwise tetrachoric correlations (i.e., $\left(\mathbf{a}_{j_{1}}^{T} \Sigma \mathbf{a}_{j_{2}}\right) /\left(\left(\mathbf{a}_{j_{1}}^{T} \Sigma \mathbf{a}_{j_{1}}+1\right)\left(\mathbf{a}_{j_{2}}^{T} \Sigma \mathbf{a}_{j_{2}}+1\right)\right)^{1 / 2}, \forall j_{1} \neq$ $j_{2}$ ). The theoretical results on the sufficient conditions turned out to be very similar to those under the linear bifactor model and extensions.

\subsection{Standard bifactor model}

Adopting the same notations as the linear bifactor model, under the probit bifactor model, the probability of a response of 1 on item $j \in\{1, \ldots, J\}$ in testlet $g_{j}$ is given by

$P\left(Y_{j}=1 \mid \eta_{0}, \eta_{1}, \ldots, \eta_{G}\right)=\Phi\left(d_{j}+a_{0} \eta_{0}+\sum_{g=1}^{G} a_{j g} \eta_{g}\right)=\Phi\left(d_{j}+a_{0} \eta_{0}+a_{j g_{j}} \eta_{g_{j}}\right)$

where, similar to the linear case, $a_{j g}=0$ for all $g \neq g_{j}$, and $\boldsymbol{\eta}=\left(\eta_{0}, \eta_{1}, \ldots, \eta_{G}\right)^{T} \sim$ $M V N(\mathbf{0}, \Sigma)$ with $\Sigma=\boldsymbol{I}_{(1+G) \times(1+G)}$. With $A$ and $\mathbf{d}$ denoting the loading matrix and the vector of intercepts, respectively, the parameter space of the probit standard bifactor model is given by $\mathfrak{P}=\{(A, \mathbf{d}) \mid$ first nonzero element in every column of $A$ is positive\}. Based on this, the definition of 
probit bifactor model identifiability is given below.

Definition 4. We say a probit bifactor model is identifiable at $(A, \mathbf{d})$ if for any other set of parameter $\left(A^{\prime}, \mathbf{d}^{\prime}\right)$ that defines the same probability distribution, it must hold that

$$
(A, \mathbf{d})=\left(A^{\prime}, \mathbf{d}^{\prime}\right)
$$

Adopting the same definitions of sets $\mathcal{H}_{1}, \mathcal{H}_{2}$, and $\mathcal{Q}_{g}$ as in section 2.1. the theorem below provides the sufficient and necessary conditions for the identifiability of dichotomous bifactor models with probit link.

Theorem 6. Under standard bifactor model with probit link, the model parameter is identifiable if and only if it satisfies one of the follow conditions.

$$
\begin{aligned}
& \text { P1 }\left|\mathcal{H}_{1}\right| \geq 3 ;\left|\mathcal{Q}_{g}\right| \geq 3 \text { for all } g=1, \ldots, G . \\
& \text { P2 }\left|\mathcal{H}_{1}\right|=2 ; \mathcal{H}_{2} \text { is non-empty; }\left|\mathcal{Q}_{g}\right| \geq 3 \text { for } g=1, \ldots, G .
\end{aligned}
$$

The interpretations of $P 1$ and $P 2$ remain the same as for the linear bifactor model in Section 2.1. In the supplementary file, a few examples are provided to illustrate how the identifiability of the probit bifactor model can be checked. 


\subsection{Extended bifactor model}

\subsection{Extended bifactor model}

With the same item response function as the standard bifactor model, the extended probit bifactor model relaxes the assumption of $\Sigma=\boldsymbol{I}_{(1+G) \times(1+G)}$ by allowing correlations among $\eta_{1}, \ldots, \eta_{G}$. The covariance matrix for $\boldsymbol{\eta}, \Sigma$, hence takes the form of $\Sigma=\left(\begin{array}{cc}1 & \mathbf{0}^{T} \\ \mathbf{0}^{T} & \boldsymbol{\Sigma}_{G}\end{array}\right)$, with $\Sigma_{G}$ positive definite with diagonal entries of 1 and no further restriction on off-diagonal entries. Under the probit extended bifactor model, the parameter space is given by $\mathfrak{P}=$ $\left\{\left(A, \mathbf{d}, \Sigma_{G}\right) \mid\right.$ first non-zero element in evey column of $A$ is positive, $\operatorname{diag}\left(\Sigma_{G}\right)=$ $1, \Sigma_{G}$ positive definite. $\}$. And the definition of probit extended bifactor model identifiability is as follows.

Definition 5. We say a probit extended bifactor model is identifiable at $\left(A, \mathbf{d}, \Sigma_{G}\right)$ if for any other set of parameters $\left(A^{\prime}, \mathbf{d}^{\prime}, \Sigma_{G}^{\prime}\right)$ that define the same probability distribution,

$$
\left(A, \mathbf{d}, \Sigma_{G}\right)=\left(A^{\prime}, \mathbf{d}^{\prime}, \Sigma_{G}^{\prime}\right)
$$

must hold.

Again, it can be shown that the sufficient conditions and necessary conditions for linear extended bifactor model still hold when the responses become binary. 
Theorem 7. Under the probit extended bifactor model, the model parameters are identifiable if one of the following set of requirements is satisfied:

E1S $\left|\mathcal{Q}_{g}\right| \geq 3$ for all $g=1, \ldots, G ;\left|\mathcal{H}_{3}\right| \geq 3$.

E2S $\left|\mathcal{Q}_{g}\right| \geq 3$ for all $g=1, \ldots, G ;\left|\mathcal{H}_{3}\right|=2 ;\left|\mathcal{H}_{2}\right| \geq 1$.

Theorem 8. Under the extended bifactor model with probit link, the model parameters are identifiable only if both conditions below are satisfied.

E1N $\left|\mathcal{Q}_{g}\right| \geq 2$ for all $g=1, \ldots, G$; and $\left|\mathcal{Q}_{g}\right| \geq 3$ for $g: \Sigma_{G}[g,-g]=\mathbf{0}$.

E2N $\left|\mathcal{H}_{3}\right| \geq 2$.

Here, the definitions of the sets $\mathcal{Q}_{g}, \mathcal{H}_{3}$, and $\mathcal{H}_{2}$ and the interpretations of the conditions remain the same as those for the linear extended bifactor model in section 2.2

\subsection{Two-tier model}

A two-tier probit model with $J$ items, $L$ main factors and $G$ testlets has the following item response function for a particular item $j$ in testlet $g_{j}$,

$P\left(Y_{j}=1 \mid \boldsymbol{\eta}\right)=\Phi\left(d_{j}+\sum_{l=1}^{L} a_{j l} \eta_{l}+\sum_{g=L+1}^{L+G} a_{j g} \eta_{g}\right)=\Phi\left(d_{j}+\sum_{l=1}^{L} a_{j l} \eta_{l}+a_{j g_{j}} \eta_{g_{j}}\right)_{3}$

where $a_{j g}=0, \forall g \neq g_{j}$. Same as the linear two-tier model, the latent traits $\boldsymbol{\eta}=\left(\boldsymbol{\eta}_{1}, \boldsymbol{\eta}_{2}\right)$, where $\boldsymbol{\eta}_{1}=\left(\eta_{1}, \ldots, \eta_{L}\right)^{T}$ and $\boldsymbol{\eta}_{2}=\left(\eta_{L+1}, \ldots, \eta_{L+G}\right)^{T}$, are 


\subsection{Two-tier model}

assumed to follow a multivariate normal distribution with mean $\mathbf{0}$ and covariance matrix $\Sigma$, which takes the form of $\left(\begin{array}{cc}\Sigma_{L} & \mathbf{0}^{T} \\ \mathbf{0}^{T} & I_{G \times G}\end{array}\right)$, with $\Sigma_{L}$ positivedefinite with diagonal elements of 1 and off-diagonal elements between -1 and 1. The parameter space for the probit two-tier model is hence given by $\mathfrak{P}=\left\{\left(A, \mathbf{d}, \Sigma_{L}\right) \mid\right.$ first non-zero element in evey column of $A$ is positive, $\operatorname{diag}\left(\Sigma_{L}\right)=$ $1, \Sigma_{L}$ positive definite\}, and the definition of probit two-tier model identifiability is as follows.

Definition 6. A probit two-tier model is identifiable at $\left(A, \mathbf{d}, \Sigma_{L}\right)$ if there is another set of parameters $\left(A^{\prime}, \mathbf{d}^{\prime}, \Sigma_{L}^{\prime}\right)$ such that $\mathbf{I}(A)=\mathbf{I}\left(A^{\prime}\right)$ and they define the same distribution, then it must hold that $\left(A, \mathbf{d}, \Sigma_{L}\right)=\left(A^{\prime}, \mathbf{d}^{\prime}, \Sigma_{L}^{\prime}\right)$.

Below we provide a set of sufficient conditions for the identifiability of the probit two-tier model.

Theorem 9. Under the probit two-tier model, suppose true parameter satisfies Condition C1 and one of Conditions T1 - T3, then the parameter is identifiable:

$T 1 S\left|\mathcal{H}_{4}\right| \geq 3, A\left[\mathcal{B}_{\mathcal{H}_{4}}, 1: L\right]$ contains an identity, where $\mathcal{B}_{\mathcal{H}_{4}}$ is the set of items that make up the testlets in $\mathcal{H}_{4}$.

T2S $\left|\mathcal{H}_{4}\right| \geq 2,\left|\mathcal{H}_{5}\right| \geq 1, A\left[\mathcal{B}_{\mathcal{H}_{4}}, 1: L\right]$ contains an identity. 
T3S A[:,1:L] contains an identity, and there exists a partition of testlets $\{1, \ldots, G\}=\mathcal{G}_{1} \cup \mathcal{G}_{2}$, such that (a) $\bar{A}_{\mathcal{G}_{1}}$ has full-column rank, and (b) $A\left[\mathcal{B}_{\mathcal{G}_{2}}, 1: L\right]$ has full-column rank.

Here, the definitions of the sets $(\mathcal{H} \mathrm{s})$ and the interpretations of the conditions remain the same as for the linear two-tier model in section 2.3,

\section{Remarks}

\subsection{Orthogonality between primary and testlet dimensions}

Discussions on the identification restriction for bifactor models can be found in Rijmen (2009), where it is pointed out that three types of identification restrictions are required.

- $G+1$ restrictions for fixing the origins of general and testlet effects.

- $G+1$ restrictions for fixing the scales of general and testlet effects.

- $G$ restrictions for dealing with the rotation issue.

By translating the restrictions to mathematical expressions, the above three conditions are equivalent to

$$
\boldsymbol{\eta} \sim N(\mathbf{0}, \Sigma), \quad \Sigma=\left(\begin{array}{cc}
1 & \mathbf{0}^{T} \\
\mathbf{0} & \Sigma_{G}
\end{array}\right),
$$




\subsection{Extensions}

with $\Sigma_{G}[g, g]=1$ for all $g \in\{1, \ldots, G\}$. However they do not provide a rigorous proof why we need the third type of restriction. Below we provide Theorem 10 to answer this question. Consider the parameter space

$$
\mathfrak{P}=\left\{\left(A, \mathbf{d}, \Sigma_{G}, \boldsymbol{\rho}, \boldsymbol{\lambda}\right) \mid \operatorname{diag}(\Sigma)=1, \Sigma \text { is positive definite }\right\},
$$

where $\Sigma=\left(\begin{array}{cc}1 & \boldsymbol{\rho}^{T} \\ \boldsymbol{\rho} & \Sigma_{G}\end{array}\right)$.

Theorem 10. The bifactor model is not identifiable at any $\left.\left(A, d, \Sigma_{G}, \boldsymbol{\rho}, \boldsymbol{\lambda}\right)\right) \in$ $\mathfrak{P}$ as defined in 4.17).

The implication of Theorem 10 is that there is no identifiable model in $\mathfrak{P}$ as defined in (4.17), that is, when the orthogonality restriction between primary and testlet dimensions is further dropped. This explains why the identification results can only be extended to correlated testlet dimensions.

\subsection{Extensions}

The results here can be extended to more general settings.

- The normality assumptions in the linear bifactor model can be removed. That is, we do not require $\eta_{g} \sim N(0,1)$ and $\epsilon_{j} \sim N\left(0, \lambda_{j}\right)$ but instead assume $\operatorname{Var}\left(\eta_{g}\right)=1$ and $\operatorname{Var}\left(\epsilon_{j}\right)=\lambda_{j}$. By checking the first and second moments, it is not hard to see that sufficient conditions in previous theorems still guarantee the identification. 
- For the ordinal probit model, each $Y_{j}$ takes values in $\left\{1, \ldots, K_{j}\right\}$ $\left(K_{j} \geq 2\right)$ and follows the following probability distribution,

$$
P\left(Y_{j}>k \mid \boldsymbol{\eta}\right)=\Phi\left(d_{j}^{(k)}+\mathbf{a}_{j}^{T} \boldsymbol{\eta}\right)
$$

for $k=1, \ldots, K_{j}-1$ with $d_{j}^{(1)} \geq d_{j}^{(2)} \ldots \geq d_{j}^{\left(K_{j}-1\right)}$. Under the same set of sufficient conditions, we can easily obtain the identifiability results.

\subsection{Connections}

Under the linear bifactor model setting, the sufficient condition in Theorem 4 given by Anderson and Rubin (1956) can be simplified in the sense that there are at least three items in each testlet, i.e. $\left|Q_{g}\right| \geq 3$ for all g. (Suppose there exists a testlet with at most two items, then it is impossible to find two disjoint submatrices of $A$ with full column rank after deleting an item within that testlet.) It can also be checked that this sufficient condition is satisfied by E1S and E2S in our Theorem 2 .

For general linear factor models, two- and three- indicator rules are two sets of simple sufficient identifiability conditions; see Bollen (1989),

- Two-indicator rules: (1) Each latent factor is related to three items; (2) Each row of $A$ has one and only one non-zero element; (3) Latent factors are uncorrelated. (4) $\epsilon$ 's are uncorrelated.

- Three-indicator rules: (1) Each latent factor is related to two items; 
(2) Each row of $A$ has one and only one non-zero element; (3) No-zero elements in $\Sigma ;(4) \epsilon$ 's are uncorrelated.

Although two- and three-indicator rules seem similar to the conditions in Theorems 1 and 2, they cannot be applied in bifactor/two-tier models. By nature, it is impossible to assume each row of $A$ has one and only one non-zero element since each item has at least two latent dimensions (general factors and testlet-specific factor). Fortunately, three items are enough for identifying the testlet effects. Thanks to the model structure, the general factor can also be identified when there is a sufficient number of testlets.

\section{Discussion}

This paper addresses the fundamental issue of identifiability of bifactor model and its extensions, under both linear model with continuous indicators and probit model with dichotomous responses. The identifiability (or nonidentifiability) of a model can be determined through easily checkable conditions. In particular, conditions $P 1$ and $P \mathscr{2}$ establish the minimum requirements that can ensure the identifiability of the standard bifactor model. For the extended bifactor model with correlated subdimensions, a set of necessary conditions $(E 1 N, E 2 N)$ and a set of sufficent conditions $(E 1 S-E 3 S)$ for parameter identifiability were proposed. Sufficient condi- 
tions for two-tier model identifiability were further presented in $C 0$ (or $C 1$ for probit model) and $T 1 S-T 3 S$. Theoretical results were able to explain underidentification phenomena observed in the existing literature. Simulation studies demonstrated the consequences on parameter estimation when the identifiability conditions were or were not met. From a practical viewpoint, these checkable identifiability conditions can guide test-developers through the design and evaluation of bifactor-type assessments.

It should be noted that, although both probit and logistic models can be applied for binary outcomes, the current identifiability results for probit models do not directly apply to item bifactor analysis with logistic parametrization, as seen in DeMars (2006), Cai (2010) and Jeon et al. (2013). When a normal distribution is assumed for the latent traits, random-effect logistic item factor models involve the convolution of Gaussian and logistic random variables. This class of models hence do not imply the same first and second moments for item responses as the probit case. Future research may look into the identifiability conditions for bifactortype models with logit link, perhaps adopting similar approaches as in San Martín et al. (2013) for two-parameter logistic item response models. The current bifactor model identification findings may also be extended to higher-order factor models Yung et al. (1999), under which latent factors 
are assumed to exhibit a hierarchical structure, with higher-order latent factors governing secondary, specific factors.

\section{Supplementary Materials}

The supplementary material contains the simulation studies, illustrative examples and technical proofs of main theoretical results.

\section{Acknowledgements}

This research was supported in part by NSF Grants DMS-2015417, SES1826540, and IIS-1633360.

\section{References}

Allman, E. S., C. Matias, J. A. Rhodes, et al. (2009). Identifiability of parameters in latent structure models with many observed variables. The Annals of Statistics 37(6A), 30993132.

Anderson, T. W. and H. Rubin (1956). Statistical inference in factor analysis. In Proceedings of the third Berkeley symposium on mathematical statistics and probability, Volume 5, pp. $111-150$.

Bollen, K. A. (1989). Structural equations with latent variables wiley. New York. 


\section{REFERENCES}

Bollen, K. A. and S. Bauldry (2010). Model identification and computer algebra. Sociological methods $\&$ research $39(2), 127-156$.

Bradlow, E. T., H. Wainer, and X. Wang (1999). A bayesian random effects model for testlets. Psychometrika 64(2), 153-168.

Cai, L. (2010). A two-tier full-information item factor analysis model with applications. Psychometrika $75(4), 581-612$.

Cai, L., K. Choi, M. Hansen, and L. Harrell (2016). Item response theory. Annual Review of Statistics and Its Application 3, 297-321.

Cai, L., J. S. Yang, and M. Hansen (2011). Generalized full-information item bifactor analysis. Psychological methods 16(3), 221.

Caspi, A., R. M. Houts, D. W. Belsky, S. J. Goldman-Mellor, H. Harrington, S. Israel, M. H. Meier, S. Ramrakha, I. Shalev, R. Poulton, et al. (2014). The p factor: one general psychopathology factor in the structure of psychiatric disorders? Clinical Psychological Science 2(2), 119-137.

Castellan, N. J. (1966). On the estimation of the tetrachoric correlation coefficient. Psychometrika $31(1), 67-73$.

Chen, F. F., A. Hayes, C. S. Carver, J.-P. Laurenceau, and Z. Zhang (2012). Modeling general and specific variance in multifaceted constructs: A comparison of the bifactor model to other approaches. Journal of personality 80(1), 219-251. 


\section{REFERENCES}

Chen, Y., X. Li, and S. Zhang (2019). Structured latent factor analysis for large-scale data: Identifiability, estimability, and their implications. Journal of the American Statistical Association, 1-15.

Chen, Y., J. Liu, G. Xu, and Z. Ying (2015). Statistical analysis of q-matrix based diagnostic classification models. Journal of the American Statistical Association 110(510), 850-866.

Christoffersson, A. (1975). Factor analysis of dichotomized variables. Psychometrika 40(1), 5-32.

DeMars, C. E. (2006). Application of the bi-factor multidimensional item response theory model to testlet-based tests. Journal of educational measurement 43(2), 145-168.

DeMars, C. E. (2012). Confirming testlet effects. Applied Psychological Measurement 36(2), $104-121$.

Eid, M., S. Krumm, T. Koch, and J. Schulze (2018). Bifactor models for predicting criteria by general and specific factors: Problems of nonidentifiability and alternative solutions. Journal of Intelligence $6(3), 42$.

Gibbons, R. D., R. D. Bock, D. Hedeker, D. J. Weiss, E. Segawa, D. K. Bhaumik, D. J. Kupfer, E. Frank, V. J. Grochocinski, and A. Stover (2007). Full-information item bifactor analysis of graded response data. Applied Psychological Measurement 31(1), 4-19.

Gibbons, R. D. and D. R. Hedeker (1992). Full-information item bi-factor analysis. Psychometrika $57(3), 423-436$. 


\section{REFERENCES}

Gibbons, R. D., A. J. Rush, and J. C. Immekus (2009). On the psychometric validity of the domains of the pdsq: An illustration of the bi-factor item response theory model. Journal of psychiatric research 43(4), 401-410.

Gignac, G. E. and M. W. Watkins (2013). Bifactor modeling and the estimation of model-based reliability in the wais-iv. Multivariate Behavioral Research 48(5), 639-662.

Green, S. and Y. Yang (2018). Empirical underidentification with the bifactor model: A case study. Educational and Psychological Measurement 78(5), 717-736.

Gu, Y. and G. Xu (2019). The sufficient and necessary condition for the identifiability and estimability of the dina model. Psychometrika 84(2), 468-483.

Holzinger, K. J. and F. Swineford (1937). The bi-factor method. Psychometrika 2(1), 41-54.

Jennrich, R. I. and P. M. Bentler (2012). Exploratory bi-factor analysis: The oblique case. Psychometrika $77(3), 442-454$.

Jeon, M., F. Rijmen, and S. Rabe-Hesketh (2013). Modeling differential item functioning using a generalization of the multiple-group bifactor model. Journal of Educational and Behavioral Statistics 38(1), 32-60.

Jöreskog, K. G. and D. Sörbom (1993). LISREL 8: Structural equation modeling with the SIMPLIS command language. Scientific Software International.

Kendall, M. G. and A. Stuart (1958). Chaps. 1-6. The advanced theory of statistics 1.

Kenny, D. A. and S. Milan (2012). Identification: A non-technical discussion of a technical 


\section{REFERENCES}

issue. Handbook of structural equation modeling, 145-163.

Kruskal, J. B. (1977). Three-way arrays: rank and uniqueness of trilinear decompositions, with application to arithmetic complexity and statistics. Linear algebra and its applications 18(2), 95-138.

Lawley, D. N. (1943). Xxiii.—on problems connected with item selection and test construction. Proceedings of the Royal Society of Edinburgh Section A: Mathematics 61(3), 273-287.

Lord, F. (1952). A theory of test scores. Psychometric monographs.

Olsson, U. (1979). Maximum likelihood estimation of the polychoric correlation coefficient. Psychometrika 44(4), 443-460.

Pearson, K. (1900). I. mathematical contributions to the theory of evolution.- - vii. on the correlation of characters not quantitatively measurable. Philosophical Transactions of the Royal Society of London. Series A, Containing Papers of a Mathematical or Physical Character $195(262-273), 1-47$.

Reilly, T. (1995). A necessary and sufficient condition for identification of confirmatory factor analysis models of factor complexity one. Sociological Methods $\&$ Research 23(4), 421-441.

Reilly, T. and R. M. O'Brien (1996). Identification of confirmatory factor analysis models of arbitrary complexity: The side-by-side rule. Sociological Methods 85 Research 24(4), 473-491.

Reise, S. P. (2012). The rediscovery of bifactor measurement models. Multivariate behavioral 


\section{REFERENCES}

research 47(5), 667-696.

Reise, S. P., J. Morizot, and R. D. Hays (2007). The role of the bifactor model in resolving dimensionality issues in health outcomes measures. Quality of Life Research 16(1), 19-31.

Rijmen, F. (2009). Efficient full information maximum likelihood estimation for multidimensional irt models. ETS Research Report Series 2009(1), i-31.

Rijmen, F. (2010). Formal relations and an empirical comparison among the bi-factor, the testlet, and a second-order multidimensional irt model. Journal of Educational Measurement $47(3), 361-372$.

San Martín, E., J.-M. Rolin, and L. M. Castro (2013). Identification of the 1pl model with guessing parameter: parametric and semi-parametric results. Psychometrika 78(2), 341379.

Thurstone, L. L. (1927). The unit of measurement in educational scales. Journal of Educational Psychology 18(8), 505.

$\mathrm{Xu}$, G. et al. (2017). Identifiability of restricted latent class models with binary responses. The Annals of Statistics 45(2), 675-707.

Xu, G. and S. Zhang (2016). Identifiability of diagnostic classification models. Psychometrika $81(3), 625-649$.

Yung, Y.-F., D. Thissen, and L. D. McLeod (1999). On the relationship between the higher-order factor model and the hierarchical factor model. Psychometrika 64(2), 113-128. 
E-mail: szhan105@illinois.edu 